\title{
ABERRANT RENAL ARTERIES
}

\author{
JOSEPH C. CERNY, M.D. \\ DANIFI, KARSCH, M.D.* \\ From the Department of Surgery, Section of Urology, \\ University of Michigan Medical Center, Ann Arbor, \\ and Henry Ford Hospital, Detroit, Michigan
}

\begin{abstract}
A case is reported of a patient with aberrant right renal artery arising at the level of the eleventh thoracic segment associated with renal artery aneurysm and systemic hypertension. Knowledge of the existence of aberrant renal arteries is important because they may be inadvertently damaged during renal surgery and their presence must be considered in evaluating a donor kidney for possible renal transplantation.
\end{abstract}

Variations in the origin and course of the renal arterial blood supply occur frequently and are of special interest to the urologist. Aberrant renal arteries may produce a variety of urologic diseases and their presence must be suspected particularly in patients with systemic hypertension or proximal ureteral obstruction.

There are various types of renal arterial aberrancies. The right and left renal arteries normally arise from the aorta within several millimeters of each other, the right originating somewhat higher. In 25 per cent of patients, significant disparity in the levels of origin may occur, in some cases as much as two vertebral segments apart. In addition, the arteries may arise from a partially dorsal or ventral aortic site rather than directly lateral.

Prehilar branching of the renal arteries is common and is usually bilaterally symmetrical. When present, the prehilar branching may occur at any point from the main renal artery, and the branch is more often superiorly than inferiorly, directed. ${ }^{1}$

Frequently, one or both kidneys are supplied by multiple renal arteries having aortic origin. Multiple arteries were seen in 28 per cent of 11,000 autopsies, and the arteries were found to arise anywhere between the eleventh thoracic and fourth lumbar vertebrae. ${ }^{2}$ Another autopsy study showed the presence of aberrant renal arteries in 49 per cent of normotensive and 80

\footnotetext{
*Present address: Chief, Urology Service, Davis-Monthan Air Force Base, Arizona, 85707
}

per cent of hypertensive patients. ${ }^{3}$ Angiographic series have shown multiple renal arteries in from 20.5 to 24 per cent of studied patients. ${ }^{4,5}$ Segmental renal arteries arising directly from the iliac, superior mesenteric, and internal spermatic arteries have been described.

This study is based on a patient in whom an aberrant right renal artery arising at the level of the eleventh thoracic segment was associated with renal artery aneurysm and systemic hypertension.

\section{Case Report}

A fifty-five-year-old white man was first noted to be hypertensive in November, 1970, at the time of routine physical examination. He was given antihypertensive medication but failure to control his hypertension necessitated further evaluation. Results of a routine intravenous pyelogram were described as normal. A transfemoral lumbar aortogram showed a normal left renal artery, but no right renal artery was seen in spite of evidence of a functional right kidney. There was no evidence of collateral circulation to the right kidney.

In March, 1971, the patient was admitted to the hospital, and physical examination was unremarkable except for hypertension (220/105) and minimal edema of the legs. No abdominal bruits were audible. Results of laboratory studies, including renal function and urinalysis, were within normal limits. Split renal function studies showed normal findings. Split renal vein 

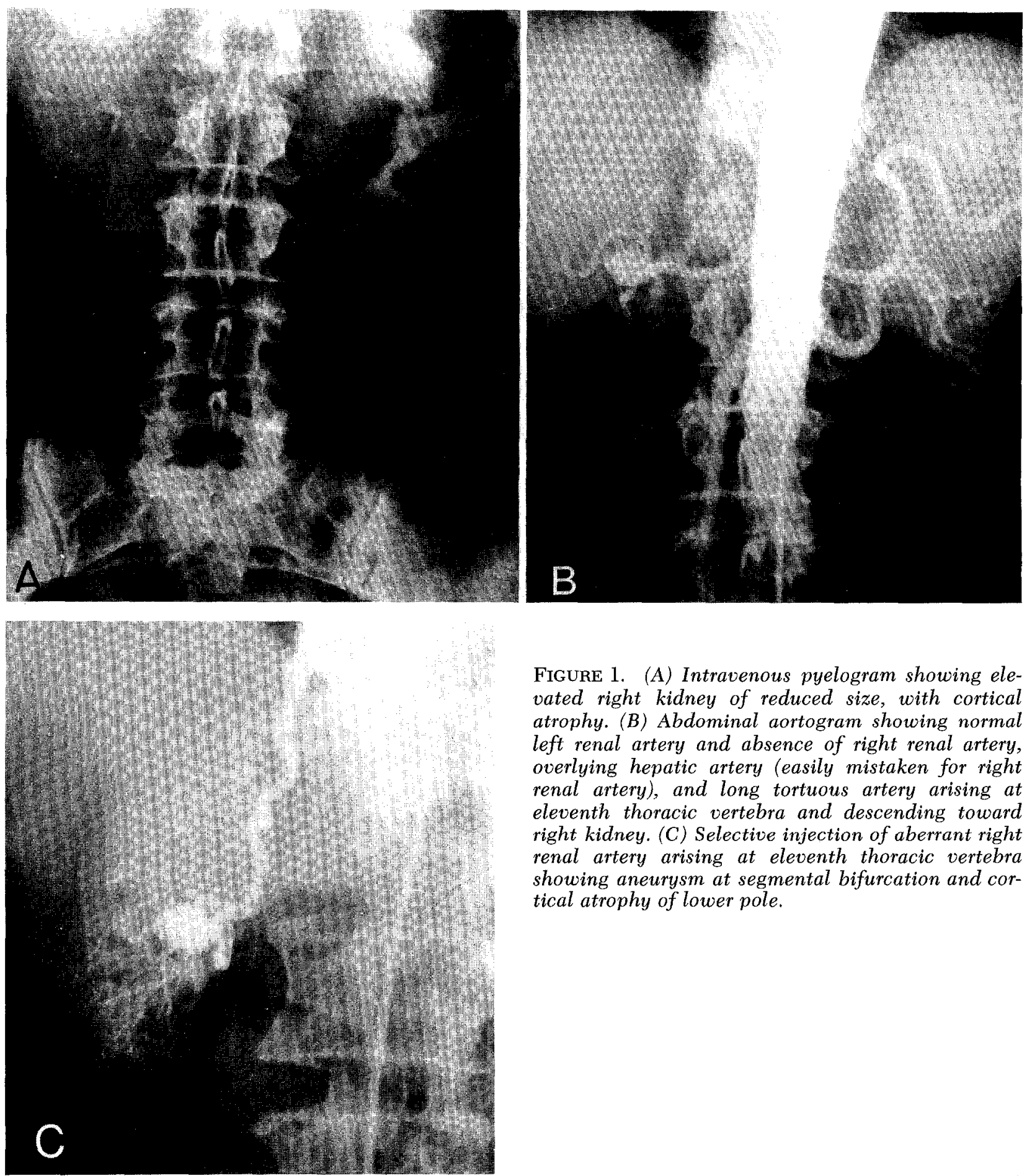

FIGURE 1. (A) Intravenous pyelogram showing elevated right kidney of reduced size, with cortical atrophy. (B) Abdominal aortogram showing normal left renal artery and absence of right renal artery, overlying hepatic artery (easily mistaken for right renal artery), and long tortuous artery arising at eleventh thoracic vertebra and descending toward right kidney. (C) Selective injection of aberrant right renal artery arising at eleventh thoracic vertebra showing aneurysm at segmental bifurcation and cortical atrophy of lower pole.

renin determinations revealed bilateral activity to be $750 \mathrm{ng}$. per $100 \mathrm{ml}$. per hour.

A hypertensive intravenous pyelogram showed a normal left kidney measuring $13 \mathrm{~cm}$. and a right kidney measuring $10.5 \mathrm{~cm}$. with loss of cortical substance in the mid- and lower portions (Fig. 1A). A renal arteriogram revealed a normal left renal artery. The right renal artery was found to arise from the lateral wall of the aorta approxi- mately $6 \mathrm{~cm}$. proximal to the celiac axis at the eleventh thoracic level (Fig. 1B and C). The artery then followed a tortuous course inferiorly toward the renal hilum, at which point a 1 by 2 $\mathrm{cm}$. aneurysm was apparent.

Transabdominal right renal exploration was performed. The right renal artery was found to arise at the eleventh thoracic level, shown angiographically, and at its entry into the renal hilum, 
an aneurysm measuring 2 by $2 \mathrm{~cm}$. in diameter was seen. The aneurysm arose at the bifurcation of the segmental arteries, and there was reduced pulsation in the vessels supplying the middle and lower portions of the kidney. The renal cortex supplied by these segmental vessels was atrophic and showed evidence of old renal infarction. The location of the aneurysm, plus the poor general quality of the renal parenchyma in the lower two thirds of the right kidney, mitigated against consideration of revascularization, and right nephrectomy was performed. The patient's postoperative course was uneventful, and at the time of discharge from the hospital, his blood pressure was maintaincd in a range of 140/90 with methyldopa (Aldomet), $250 \mathrm{mg}$. given orally three times a day.

Pathologic examination of the surgical specimen revealed a dissecting renal artery aneurysm secondary to atherosclerosis. Portions of the kidney supplied by the involved segmental branches showed severe ischemia and chronic pyelonephritis.

Six months follow-up has shown that the patient requires methyldopa, $250 \mathrm{mg}$. three times a day to maintain a blood pressure range of $140 / 90$. He is otherwise well.

\section{Comment}

This patient's clinical course of aberrant renal artery associated with renal ischemia and hyper- tension is not unusual. As previously stated, Marshall's ${ }^{3}$ autopsy series has documented a significantly higher incidence of aberrant renal arteries in hypertensive than in normotensive patients, and he has suggested a causal relationship secondary to renal ischemia.

Other lesions related to aberrant renal arteries and of interest to urologists are (1) upper ureteral or ureteral-pelvic junction obstruction produced by vessels to the lower pole of the kidney, and (2) chronic pyelonephritis, presumably the result of aberrant vascular ischemia or ureteral outflow obstruction as described. In addition, an awareness of renal arterial variations is mandatory for any surgeon, since inadvertent laceration of such vessels at the time of renal surgery may result in severe hemorrhage and/or infarction of renal parenchyma. Further, the suitability of a donor kidney for related or cadaveric renal transplantation may be jeopardized by the presence of aberrant arterial supply.

The variations in renal arterial supply described are best understood when the embryology of the renal vasculature is considered (Fig. 2). In the human embryo, the mesonephros extends from the eighth cervical to the twelfth thoracic vertebrae and receives its blood supply from the "rete arteriosum urogenitale," a series of approximately 20 segmental branches from the lateral aorta. ${ }^{2}$ Normally, these vessels involute as the metanephros or mature embryonic kidney develops with its lower aortic blood supply.

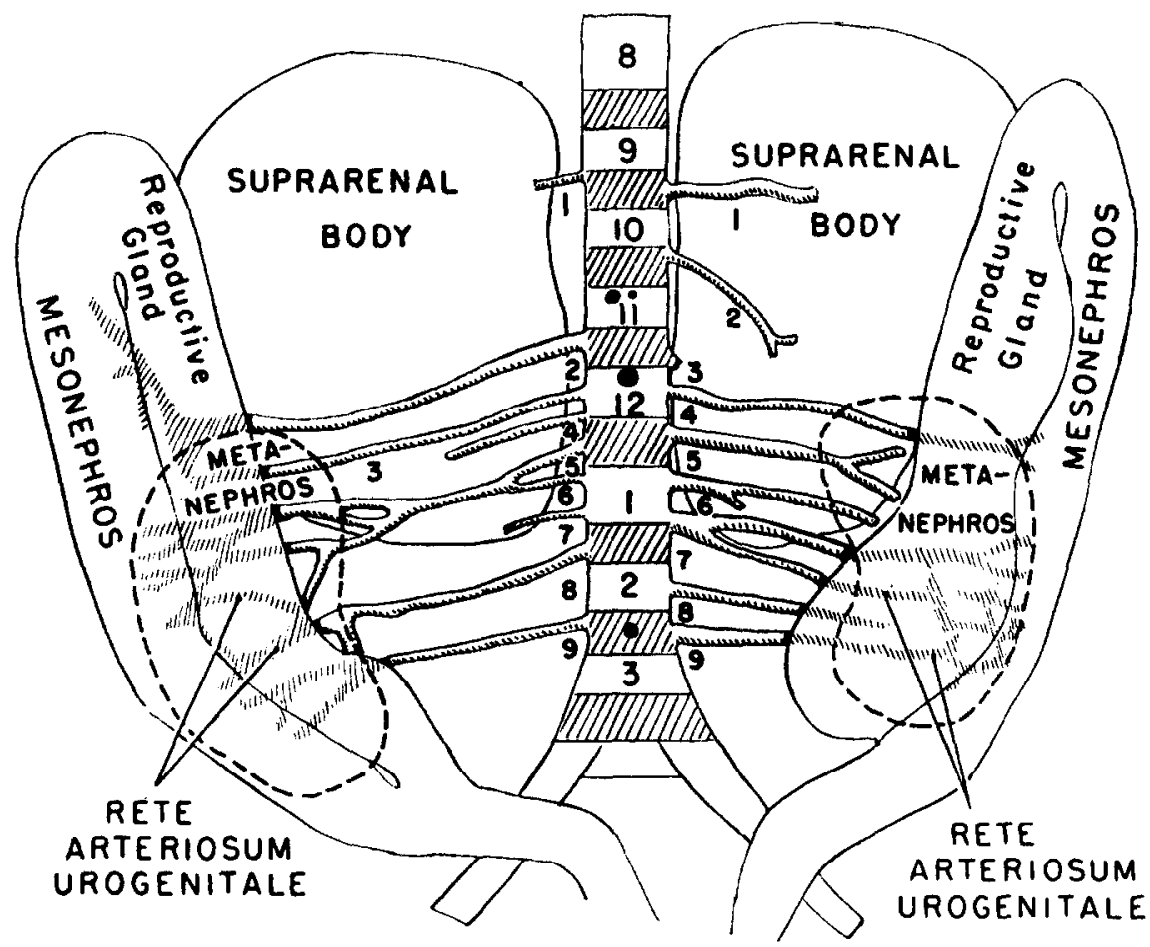

Figure 2. Diagram showing mesonephric blood supply "rete arteriosum urogenitale," and its relationship to eventual metanephros; persistence of mesonephric vessels may account for aberrant arterial supply of mature kidney. 
Persistence of certain of the more cephalad mesonephros vessels, however, may result in the arterial abnormalities described.

Review of the literature has shown that high aortic origin of a single renal artery, as described in our case, is rare; only two previous reports have been found. ${ }^{6,7}$ Also in our case knowledge of the possibility of the origin of a single renal artery as high as the eleventh thoracic vertebra is important to the angiographer, since this level may not be visualized during routine abdominal aortography.

\section{References}

1. DAvis, G. D.: Variations in renal blood supply, in Kincaid, O. W., and Davis, G. D.: Renal Angiography, Chicago, Year Book Medical Publishers, 1966, pp. 83-87.

2. MERKLIN, R. J., and Michels, N. A.: The variant renal and suprarenal blood supply with data on the inferior phrenic, ureteral and gonadal arteries: A statistical analysis based on 185 dissections and reviews of the literature, J. Int. Coll. Surg. 29: 41 (1958).

3. Marshall, A. G.: Aberrant renal arteries and hypertension, Lancet 2: 701 (1951).

4. EDSMAN, G.: Angionephrography and suprarenal angiography: A roentgenologic study of the normal kidney, expansive renal and suprarenal lesions and renal aneurysms, Acta Radiol. (suppl.) 155: 1 (1957).

5. BorJSEN, E.: Angiographic studies of the anatomy of single and multiple renal arteries, ibid (suppl.) 183: 1 (1959).

Henry Ford Hospital 2799 West Grand Boulevard Detroit, Michigan 48202 (DR. CERNY)
6. Doppman, J.: An ectopic renal artery, Br. J. Radiol. 40: $312(1967)$.

7. Tegtmeyer, C. J., and Stanton, C. W.: Thoracic origin of a single renal artery, J.A.M.A. 209: 1223 (1969). 